\title{
Power deposition behavior of high-density transient hydrogen plasma on tungsten in Magnum-PSI
}

\section{Citation for published version (APA):}

Li, Y., Morgan, T. W., van den Berg-Stolp, J., Genuit, J. W., De Temmerman, G., Hoefnagels, J. P. M., van Dommelen, J. A. W., Verbeken, K., \& Geers, M. G. D. (2021). Power deposition behavior of high-density transient hydrogen plasma on tungsten in Magnum-PSI. Plasma Physics and Controlled Fusion, 63(8), [085016]. https://doi.org/10.1088/1361-6587/ac0bc9

\section{Document license:}

TAVERNE

DOI:

10.1088/1361-6587/ac0bc9

Document status and date:

Published: 01/08/2021

\section{Document Version:}

Publisher's PDF, also known as Version of Record (includes final page, issue and volume numbers)

\section{Please check the document version of this publication:}

- A submitted manuscript is the version of the article upon submission and before peer-review. There can be important differences between the submitted version and the official published version of record. People interested in the research are advised to contact the author for the final version of the publication, or visit the $\mathrm{DOI}$ to the publisher's website.

- The final author version and the galley proof are versions of the publication after peer review.

- The final published version features the final layout of the paper including the volume, issue and page numbers.

Link to publication

\section{General rights}

Copyright and moral rights for the publications made accessible in the public portal are retained by the authors and/or other copyright owners and it is a condition of accessing publications that users recognise and abide by the legal requirements associated with these rights.

- Users may download and print one copy of any publication from the public portal for the purpose of private study or research.

- You may not further distribute the material or use it for any profit-making activity or commercial gain

- You may freely distribute the URL identifying the publication in the public portal.

If the publication is distributed under the terms of Article 25fa of the Dutch Copyright Act, indicated by the "Taverne" license above, please follow below link for the End User Agreement:

www.tue.nl/taverne

Take down policy

If you believe that this document breaches copyright please contact us at:

openaccess@tue.nl

providing details and we will investigate your claim. 
PAPER

Power deposition behavior of high-density transient hydrogen plasma on tungsten in Magnum-PSI

To cite this article: Y Li et al 2021 Plasma Phys. Control. Fusion 63085016

View the article online for updates and enhancements.

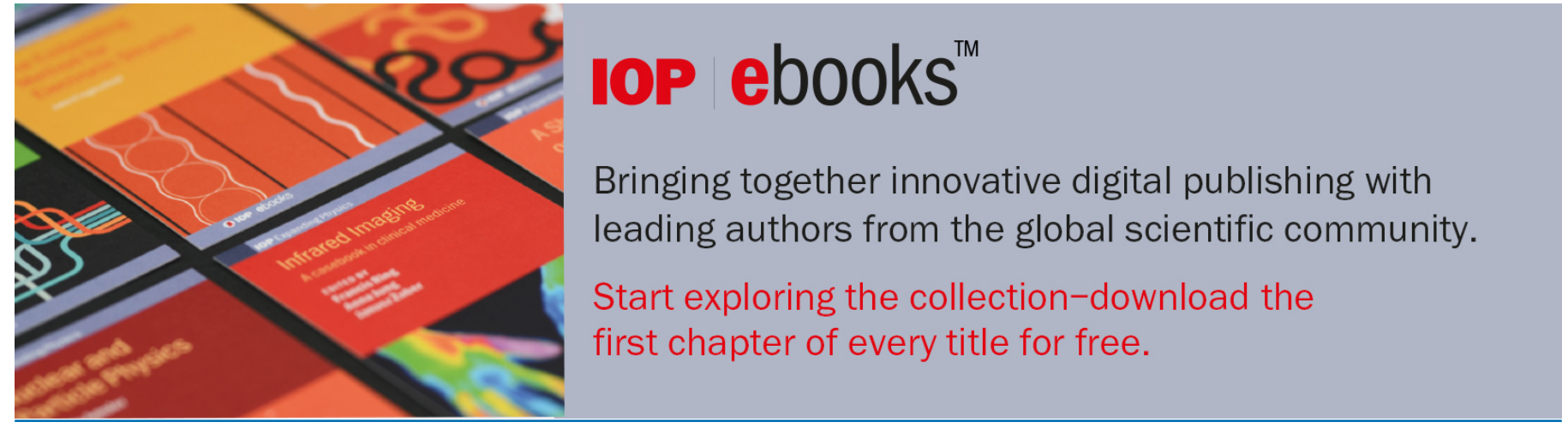

This content was downloaded from IP address 131.155 .153 .98 on $05 / 10 / 2021$ at 08:56 


\title{
Power deposition behavior of high-density transient hydrogen plasma on tungsten in Magnum-PSI
}

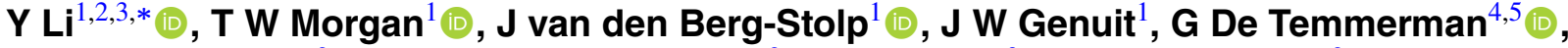 J P M Hoefnagels ${ }^{2}$, J A W van Dommelen ${ }^{2}, K_{\text {Verbeken }}^{3}$ and M G D Geers ${ }^{2}$}

\author{
${ }^{1}$ DIFFER-Dutch Institute for Fundamental Energy Research, De Zaale 20, 5612 AJ Eindhoven, The \\ Netherlands \\ ${ }^{2}$ Department of Mechanical Engineering, Eindhoven University of Technology, PO Box 513, 5600 MB, \\ Eindhoven, The Netherlands \\ ${ }^{3}$ Department of Materials, Textiles and Chemical Engineering, Ghent University (UGent), \\ Technologiepark 46, B-9052 Ghent, Belgium \\ ${ }^{4}$ Zenon Research, Paris F-75006, France \\ ${ }^{5}$ MINES ParisTech, University PSL, Institute of Higher Studies for Innovation and Entrepreneurship \\ (IHEIE), Paris 75006, France
}

E-mail: Y.Li@differ.nl

Received 24 February 2021, revised 22 May 2021

Accepted for publication 16 June 2021

Published 30 June 2021

\begin{abstract}
The lifetime of plasma-facing components (PFCs) will have a strong influence on the efficiency and viability of future fusion power plants. However, the PFCs suffer from thermal stresses and physical sputtering induced by edge-localized modes (ELMs). ELMs in future fusion devices are expected to occur with a high plasma density compared to current day devices such that coupling of recycling neutrals and plasma ions will be strong. Because of the scale hierarchy of future fusion devices compared to the present ones, the influence of this coupling is difficult to predict. Here, we investigate the ELM-like hydrogen plasma induced heat loads on tungsten in the linear device Magnum-PSI, producing $\sim 1 \mathrm{~ms}$ plasma pulses with electron densities up to $3.5 \times 10^{21} \mathrm{~m}^{-3}$. A combination of time-resolved Thomson scattering and coherent Thomson scattering was used to acquire plasma parameters in front of the target. Moreover, a fast infrared camera coupled to finite element thermal analyses allowed to determine the deposited heat loads on the target. We found a significant inconsistency between the plasma power calculated with a conventional collisionless sheath model and the absorbed power by the target. Moreover, plasma stagnation upstream and plasma cooling downstream were observed during the pulses. The observations are explained based on ionization and elastic collisions between the recycling neutrals and plasma ions. The results highlight the impact of plasma-neutral interaction on the power deposition behavior of ELM-like hydrogen plasma on tungsten.
\end{abstract}

Keywords: edge-localized modes, neutrals recycling, plasma flow, sheath physics, nuclear fusion

(Some figures may appear in colour only in the online journal)

\section{Introduction}

The problems arising from plasma-surface interactions hamper the realization of a viable fusion reactor. Tungsten

\footnotetext{
Author to whom any correspondence should be addressed
}

is the leading material to handle the plasma exhaust challenge in ITER and future fusion devices $[1,2]$. However, the explosive heat and particle outburst during edge-localized modes (ELMs), which are magnetohydrodynamic instabilities occurring at the edge of $\mathrm{H}$-mode plasmas [3, 4], degrade the structural integrity of the tungsten plasma-facing components 


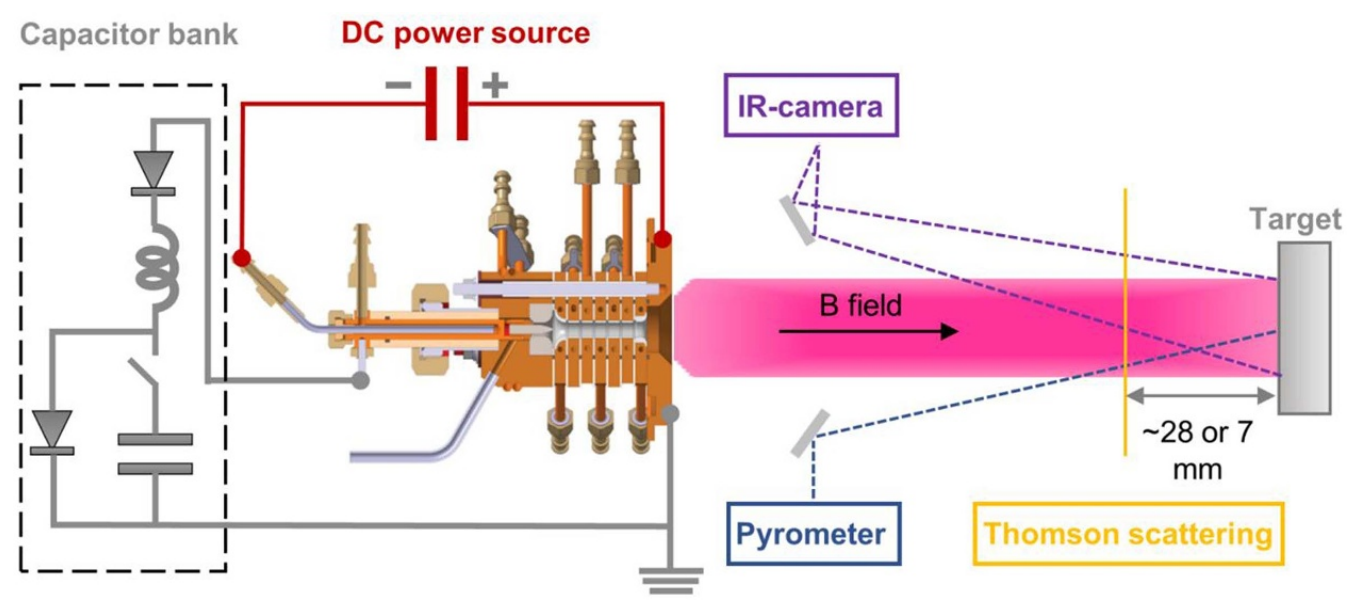

Figure 1. Sketch of the experimental setup. The pulsed hydrogen plasma is generated by the pulsed source system (left), directed to a water-cooled tungsten target by a strong axial magnetic field. Plasma parameters are monitored by Thomson scattering at a distance of $\sim 28 \mathrm{~mm}$ or $\sim 7 \mathrm{~mm}$ in front of the target. The surface temperature of the target is measured by a combination of a pyrometer and an infrared camera.

(PFCs) and introduce tungsten impurities into the plasma [5-10]. Understanding such extreme plasma surface interactions is critical in determining the operational limits of PFCs and instrumental for the operation of future fusion devices, therefore motivating extensive experimental and numerical work in recent years.

A crucial task is to understand the ELM induced heat load on the PFCs. For tokamaks, the latest multi-machine scaling links pedestal top plasma quantities to the peak ELM energy fluence on the outer divertor target plates [11]. Although this scaling seems to work within the adopted dataset, near-target plasma-neutral interactions are not fully considered, which undermines its predictability when extrapolating to ITER, for which the divertor conditions and particle fluxes will be significantly different. In the DIII-D tokamak, particle recycling was shown to cause a significant increase in electron density and ion flux in the divertor during ELMs [12]. ELM sputtered impurities were shown to cool the plasma in the JET tokamak, leading to temporary plasma detachment [13]. In the linear plasma generator Pilot-PSI, which produces ELM-like hydrogen plasmas, target outgassing was suggested to shield the tungsten surface from the intense plasma heat load [14]. These works underline the importance of near-target plasma-neutral interactions in understanding the ELM induced heat load on PFCs, motivating the current study.

The present study aims to improve the understanding of the ELM-like hydrogen plasma induced heat load on tungsten. Utilizing the unique pulsed plasma system of MagnumPSI, which generates ELM-like transient events [15], a twosided approach was taken. On the one hand, near-target plasma parameters were measured by a combination of time-resolved Thomson scattering (TS) and coherent Thomson scattering (CTS) to assess the plasma heat loads. On the other hand, fast infrared thermography coupled to finite element thermal analyses was performed to determine the deposited heat loads on the target. The comparison of these two quantities provides novel insights into the plasma-surface interactions.

\section{Experimental and numerical procedures}

\subsection{Plasma exposure}

The experimental setup of the linear plasma generator Magnum-PSI is schematically illustrated in figure 1. In steadystate, the machine produces a low-temperature high-density plasma with a cascaded arc source, which is relevant to the conditions expected in the ITER divertor [16, 17]. In parallel, a modular capacitor bank system is connected to the plasma source and used to transiently generate higher temperatures and densities on top of the steady-state plasma at a frequency of $5 \mathrm{~Hz}$ by dissipating the stored energy in the capacitors $[15,18]$. The plasma expands supersonically into the vacuum vessel and is radially confined by a strong axial magnetic field. A three-stage differentially pumped vacuum system is used to keep the neutral pressure in the target chamber below $1 \mathrm{~Pa}$ [19]. An electrically isolated tungsten target measuring $20 \times 20 \times 3 \mathrm{~mm}$ was clamped to a watercooled copper holder with a GRAFOIL ${ }^{\circledR}$ layer in between using a titanium-zirconium-molybdenum alloy clamping ring. The plasma discharge parameters are summarized in table 1 .

\subsection{In-situ diagnostics}

The electron density $\left(n_{\mathrm{e}}\right)$ and electron temperature $\left(T_{\mathrm{e}}\right)$ of the plasmas were measured by a TS system at a distance of $\sim 28 \mathrm{~mm}$ or $\sim 7 \mathrm{~mm}$ in front of the target. The TS system [20] uses a Spectron laser (model SL 8354) that operates at the second harmonic $532 \mathrm{~nm}$ and a $10 \mathrm{~Hz}$ repetition rate. The laser has a diameter of $9.5 \mathrm{~mm}$, a pulse width of $12 \mathrm{~ns}$ and delivers 0.7 Joule per pulse energy. With a scattering angle between $84.7^{\circ}$ and $95.3^{\circ}$ along the full laser chord length, the TS light is imaged onto a linear fiber array of 59 fibers (CeramOptek UV400/424P) by a lens (AF Nikkor $85 \mathrm{~mm} \mathrm{f} / 1.8 \mathrm{D}$ ) at the target chamber position or another lens (AF DC-Nikkor $135 \mathrm{~mm}$ $\mathrm{f} / 2 \mathrm{D}$ ) at the source chamber position. Finally, the TS light is 
Table 1. Hydrogen plasma discharge parameters.

\begin{tabular}{|c|c|c|c|c|c|}
\hline $\begin{array}{l}\text { Discharge } \\
\text { mode }\end{array}$ & $\begin{array}{c}\mathrm{H}_{2} \text { gas } \\
\text { flow, }(\mathrm{slm})\end{array}$ & $\begin{array}{l}\text { Cathode } \\
\text { DC, (A) }\end{array}$ & $\begin{array}{l}\text { B-field, } \\
\text { (T) }\end{array}$ & $\begin{array}{c}\text { Capacitor } \\
\text { bank stored } \\
\text { energy, }(\mathrm{J})\end{array}$ & $\begin{array}{l}\text { Reference } \\
\text { number }\end{array}$ \\
\hline \multirow{13}{*}{$\begin{array}{l}\text { Steady-state } \\
+ \\
\text { transient }\end{array}$} & \multirow[t]{4}{*}{14} & \multirow[t]{9}{*}{150} & \multirow[t]{9}{*}{1.2} & 18.75 & 1 \\
\hline & & & & 42.19 & 2 \\
\hline & & & & 75 & 3 \\
\hline & & & & 117 & 4 \\
\hline & \multirow[t]{5}{*}{12} & & & 75 & 5 \\
\hline & & & & 108 & 6 \\
\hline & & & & 147 & 7 \\
\hline & & & & 192 & 8 \\
\hline & & & & 243 & 9 \\
\hline & \multirow[t]{4}{*}{9.6} & \multirow[t]{4}{*}{180} & & 75 & 10 \\
\hline & & & & 108 & 11 \\
\hline & & & & 147 & 12 \\
\hline & & & & 192 & 13 \\
\hline \multirow[t]{6}{*}{ Steady-state } & \multirow[t]{6}{*}{8} & \multirow[t]{6}{*}{150} & 1.5 & 0 & 14 \\
\hline & & & 1.3 & & 15 \\
\hline & & & 1.1 & & 16 \\
\hline & & & 0.9 & & 17 \\
\hline & & & 0.7 & & 18 \\
\hline & & & 0.5 & & 19 \\
\hline
\end{tabular}

collected by a high-etendue transmission grating spectrometer, which is equipped with an intensified charge-coupled device camera (PI-max 1300, Princeton Instruments). By increasing the delay in the triggering of the TS system with respect to the pulsed plasma generation system by $200 \mu$ s each time, a time-resolved (stroboscopic) measurement of these parameters was built up over 300 identical plasma pulses. This approach is described in more detail in [17]. Additionally, CTS was performed to acquire the axial plasma flow velocity in the beam center at a distance of $\sim 28 \mathrm{~mm}$ in front of the target with a stroboscopic time of $120 \mu \mathrm{s}$. The CTS system uses a $1064 \mathrm{~nm}$ laser (Continuum DLS 8000, $20 \mathrm{~Hz}, 7$ ns full width at half maximum (FWHM)), which is coupled into the TS laser beamline by using a dichroic mirror. The CTS light is collected at a scattering angle of $13^{\circ}$ by a spectrometer which is equipped with an Echelle grating operating at the blaze angle and a LIVAR M506 EB-camera (EBABS technology, Electron Bombarded Active Pixel Sensor) [21, 22]. Note that the TS and CTS measurements were performed separately under the same discharge conditions. By monitoring the current-voltage characteristics of the plasma source, the floating potential and the surface temperature of the target, the plasma discharges were found to be highly reproducible.

The surface temperature of the target was monitored using a combination of a pyrometer and an infrared camera. Targeting the plasma beam center, a multi-wavelength (250 channels) pyrometer (FAR Associates ${ }^{\circledR}$ FMPI) operating in the spectral range of $1.1-1.7 \mu \mathrm{m}$ with a spot size of $\sim 3 \mathrm{~mm}$ was used. Sufficient accuracy was achieved regardless of the strong hydrogen absorption and emission lines (see appendix A in [23]). Furthermore, the 2D surface temperature of the target was recorded by a fast-framing infrared camera $\left(\right.$ FLIR $^{\circledR}$
SC7500MB) in the wavelength range 3.97-4.01 $\mu \mathrm{m}$ and at a frequency of $\sim 5 \mathrm{kHz}$. The spatial resolution (pixel size) of the infrared camera is estimated at $\sim 300 \mu \mathrm{m}$. It was verified that no hydrogen emission line was present in the spectral range of the infra-red camera. Therefore, the measured intensity solely originates from the black-body radiation of the target. By accounting for temperature-, wavelength-, and surfacedependent emissivity of tungsten, as described in [24], the surface temperature was determined. The total power delivered to the target by the plasma was obtained from the cooling water temperature rise via calorimetry.

\subsection{Numerical thermal analysis}

The three-dimensional heat equation was solved using the finite element method (FEM) in MSC.Marc/Mentat ${ }^{\circledR}$, to obtain the target temperature response. In this paper, the target was discretized into 32000 eight-node hexahedron finite elements. Temperature-dependent thermal conductivity and heat capacity of tungsten were taken from [25, 26], respectively. The current pulse through the source consists of two modes. The rise mode is described by the first quarter of a sine wave, whereas the decay mode follows an exponential decay [15]. Therefore, the heat flux profile in the simulation was prescribed to take this functional form. The magnitude of the peak heat flux, the period of the rise mode, and the e-folding time of the decay mode were fitted to the experimental data, as will be shown in figure 3(c). The spatial profile of the heat flux was obtained from the target surface temperature distribution (10 mm FWHM), which only varied slightly during the pulse. It was verified that this variation has negligible influence on the obtained peak heat flux. The FEM analysis procedure has been elaborated in a previous study [24], the accuracy of which is below $15 \%$.

\section{Heat flux measurements}

\subsection{Steady-state heat flux}

The collisionless sheath model provides the heat flux $q$ as a function of the electron density $n_{\mathrm{e}}^{\mathrm{TS}}$ and the electron temperature $T_{\mathrm{e}}^{\mathrm{TS}}$ at the sheath edge as follows [27]:

$$
q=0.5 n_{\mathrm{e}}^{\mathrm{TS}} \gamma k_{\mathrm{B}} T_{\mathrm{e}}^{\mathrm{TS}} \underbrace{\left[\left(k_{\mathrm{B}} T_{\mathrm{e}}^{\mathrm{TS}}+\frac{5}{3} k_{\mathrm{B}} T_{\mathrm{i}}^{\mathrm{TS}}\right) / m_{\mathrm{i}}\right]^{\frac{1}{2}}}_{c_{s e}},
$$

where $k_{\mathrm{B}}$ is the Boltzmann constant and $\gamma$ is the sheath heat transmission coefficient $\left(\sim 7\right.$ when $T_{\mathrm{e}}>5 \mathrm{eV}$, as detailed in appendix $\mathrm{A}) . c_{\mathrm{se}}$ is the ion velocity at the sheath edge, which is taken to be the ion sound speed (Bohm criterion) [28]. In calculating $c_{\mathrm{se}}, T_{\mathrm{e}}^{\mathrm{TS}}=T_{\mathrm{i}}^{\mathrm{TS}}$ is assumed, as confirmed by CTS measurements. Under the assumption of a collisionless and isothermal plasma, the upstream plasma Mach number is 0.5 [29]. Hence, the coefficient 0.5 in equation (1) results from the particle flux conservation of the ions. 

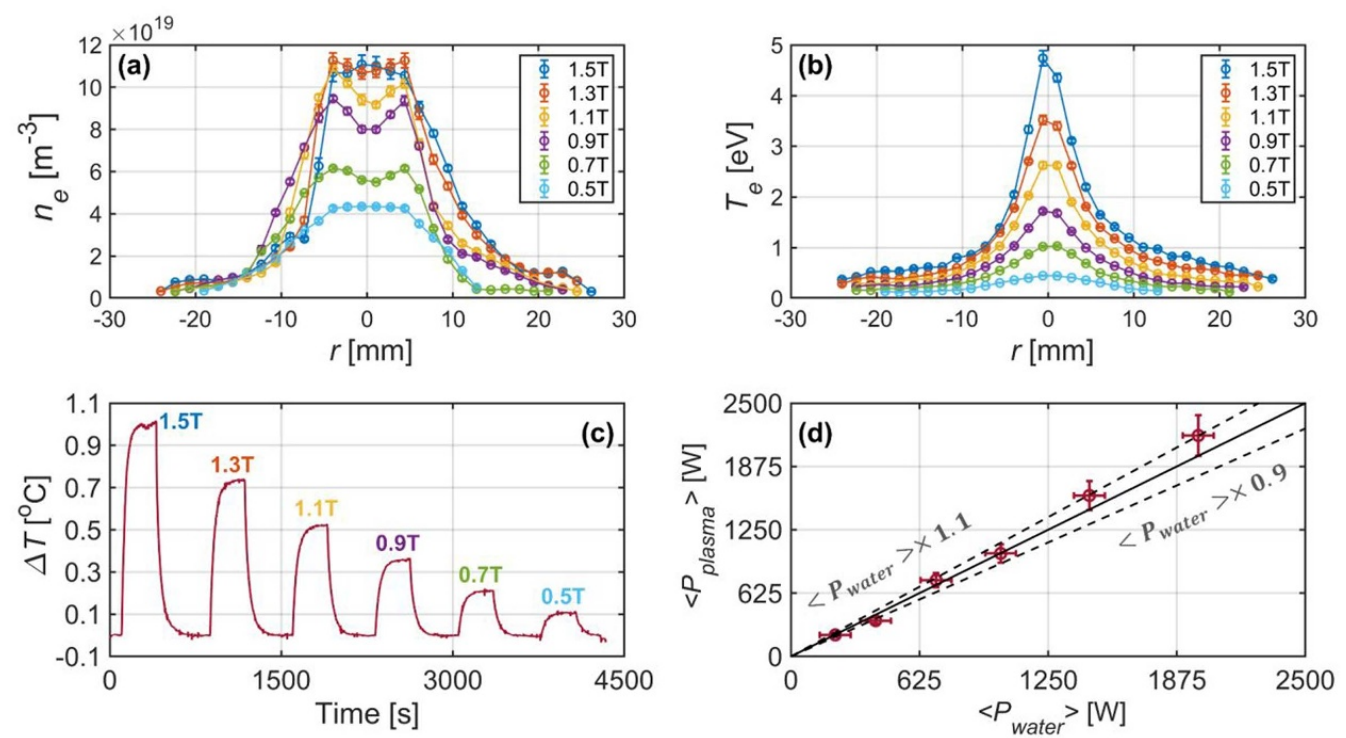

Figure 2. Accuracy analysis of $\gamma$ in equation (1) for steady-state $\mathrm{H}$ plasmas. Figures (a) and (b) show the spatial distribution of, respectively, $n_{\mathrm{e}}$ and $T_{\mathrm{e}}$ for the indicated magnetic fields. (c) The corresponding cooling water temperature evolution. (d) The integrated plasma heating power vs. the water-cooling power of the target.
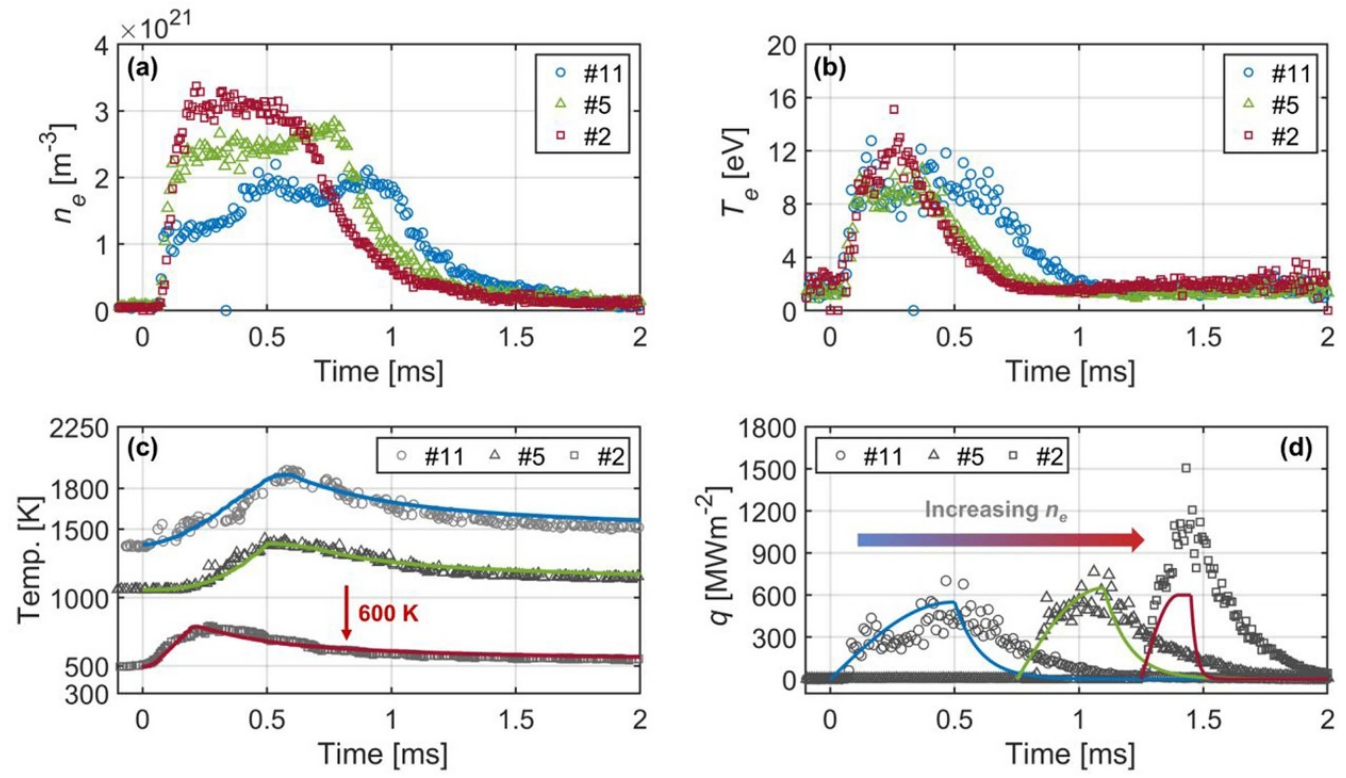

Figure 3. Heat fluxes of the pulsed plasma. Figures (a) and (b) are representative time-resolved TS measurements. (c) The surface temperature profiles, measured independently with infrared imaging, as symbols and the optimal FEM simulations as lines. (d) The calculated heat fluxes using equation (1) as symbols and FEM as lines. The legend refers to the discharge number in table 1.

The accuracy of $\gamma$ is assessed next. For steady-state hydrogen plasmas in Magnum-PSI, our previous study showed that the collisionless and isothermal assumptions in equation (1) are reasonable when $n_{\mathrm{e}}<2 \times 10^{20} \mathrm{~m}^{-3}$ [22]. Therefore, by keeping $n_{\mathrm{e}}$ below $2 \times 10^{20} \mathrm{~m}^{-3}$ and varying $T_{\mathrm{e}}$ in the range of $0.5-5 \mathrm{eV}$, where $\gamma$ is a strong a function of $T_{\mathrm{e}}$, the accuracy of $\gamma$ was evaluated under these conditions. The spatial distribution of $n_{\mathrm{e}}$ and $T_{\mathrm{e}}$ of these discharges are shown in figures 2(a) and (b). Then, $q$ in equation (1) was integrated over the plasma beam to obtain the total heating power on the target. The cooling water temperature evolution resulting from these steady-state conditions is plotted in figure 2(c) and from this the total power on the target was determined by calorimetry. Comparing the power determined using equation (1) to the power determined from calorimetry, the difference is found to be approximately within $10 \%$. Equation (1) is therefore used to calculate the plasma heat flux in this study, which will be referred as $q_{\text {collisionless }}$ thereafter.

\subsection{Transient heat flux}

Figure 3 demonstrates the two-fold approach to determine the heat fluxes of the pulsed plasma. Three typical time-resolved 


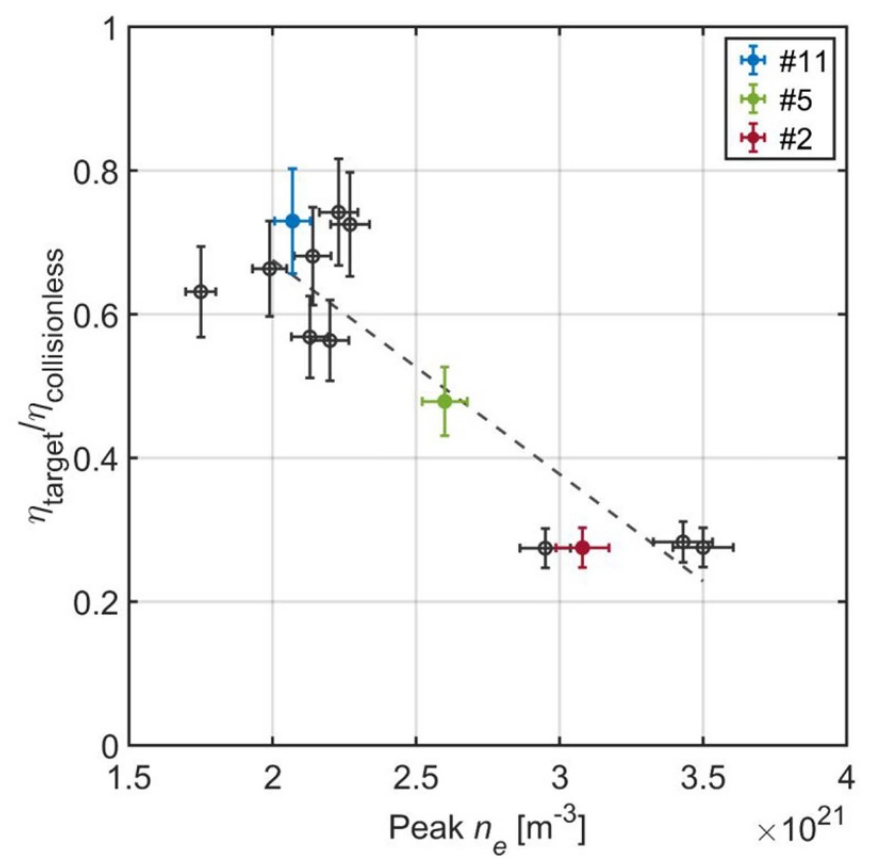

Figure 4. The energy density ratio $\frac{\eta_{\text {target }}}{\eta_{\text {collisionless }}}$ vs. the peak electron density $n_{\mathrm{e}}$. The dashed line denotes the linear regression of the dataset. The colored symbols refer to the measurements in figure 3 .

TS measurements in the center of the plasma beam are shown in figures 3(a) and (b), covering the studied peak electron density range. The legend refers to the discharge number in table 1. The $n_{\mathrm{e}}$ profile displays an approximately flat top, the value of which increases with the hydrogen gas flow in the plasma source. The peak $T_{\mathrm{e}}$ is approximately $12 \mathrm{eV}$. Using equation (1), the plasma heat fluxes are calculated in figure 3(d). For clarity, discharge \#5 and discharge \#2 have been shifted along the time axis for $0.75 \mathrm{~ms}$ and $1.25 \mathrm{~ms}$, respectively. On the other hand, figure 3(c) displays the surface temperature profiles, measured with infrared imaging (symbols) and through the optimized FEM simulations (lines). Discharge \#2 has been shifted down by $600 \mathrm{~K}$ for clarity, as indicated by the red arrow. The FEM input heat fluxes are shown in figure 3(d) as lines, representing the absorbed plasma and neutral fluxes by the target. Clearly, each heat flux determined by the FEM analysis is shorter in duration than that calculated from equation (1). The higher the peak $n_{\mathrm{e}}$, the shorter the duration. Moreover, it is remarkable that the peak heat flux saturates at the highest $n_{\mathrm{e}}$, as indicated by the flat-top heat flux profile of discharge \#2. Discharge \#3 and \#4 exhibit similar heat flux profiles as discharge \#2. Since the area under the heat flux profile is the energy density $\eta$, figure 3(d) therefore reveals a significant energy inconsistency between that calculated by the collisionless sheath model and that absorbed by the target for the pulsed plasma. As summarized in figure 4, the energy density ratio $\frac{\eta_{\text {target }}}{\eta_{\text {collisionless }}}$ decreases with the peak (flat-top) $n_{\mathrm{e}}$. This $n_{\mathrm{e}}$ dependence suggests that this energy inconsistency is related to plasma-surface interaction, as will be explored further.

\section{Flow velocity measurements}

To help understand the preceding results, the plasma flow velocity at the plasma beam center was measured by CTS at a distance of $\sim 28 \mathrm{~mm}$ in front of the target for discharge \#1-3, which reveals a strong heat flux reduction and energy dissipation. Figure 5(a) displays the evolution of the Mach numbers during the plasma pulses, obtained by dividing the measured flow velocity (inset) with its corresponding acoustic velocity $c_{\mathrm{se}}$. As the plasma pulse evolves, the Mach number first decreases strongly then recovers in the end. Compared to the steady-state hydrogen plasmas in Magnum-PSI, the Mach number of which has been measured to be $0.2-0.4$ [22], it is remarkable that the Mach number is close to zero in the middle of each pulse. In other words, the plasma is temporarily stagnant, as indicated by the grey patch in figure 5(a).

The stagnation of the plasma results from a combination of elastic collisions and ionization between the plasma ions and recycling neutrals. While the former is prevailing for steadystate H plasmas in Magnum-PSI [22], it alone cannot entail the plasma stagnation as it requires a flowing plasma to thermalize as neutrals at the target. Therefore, we consider the role of ionization in the plasma flow behavior. As sketched in figure 5(b), we postulate that a large fraction of neutrals desorbing from the target is ionized near the target. They are then driven to the target and thermalize therein, after which they desorb and are ionized again in the plasma. As a result, the near-target plasma becomes partially self-sustained; that is, a large fraction of hydrogen particles is recycled between the target and the neartarget plasma. Given particle conservation, this corresponds to a greatly reduced plasma flow velocity at the upstream position. Alternatively, considering momentum conservation, the newly created ions constitute an effective drag to the plasma when they are brought to the local speed of the fluid [27]. Consequently, in combination with elastic collisions, the plasma flow is limited to a thin layer near the target, as illustrated in figure 5(b). Because the thickness of this near-target ionization layer is of the order of the ionization mean-free-path, which is less than $10 \mathrm{~mm}$ during the plasma pulse (as will be shown in figure 7), and since the CTS system is placed $\sim 28 \mathrm{~mm}$ in front of the target, the CTS system in fact measures the plasma stagnation region during the pulse. It is noted that this flow behavior is reminiscent of the high recycling divertor conditions in tokamak experiments where the plasma flux from the core plasma to the scrape-off layer is much smaller than the plasma flux due to ionization in the divertor region [30]. Taken together, the stagnation of the plasma reveals significant ionization between the plasma ions and recycling neutrals, which will be further analyzed in section 5.1.

\section{Discussion}

The heat flux reduction in figure 3(d) and energy dissipation in figure 4 will be discussed first and subsequently, the obtained insights will be projected onto ITER in section 5.3. 

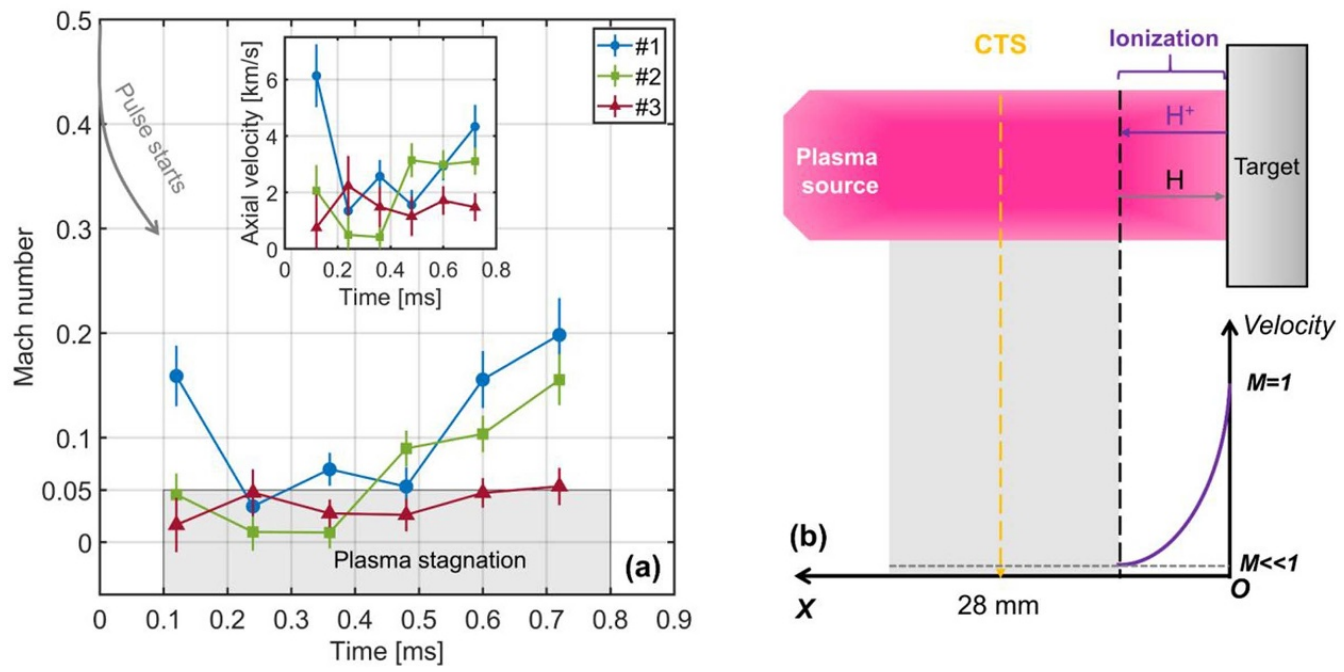

Figure 5. (a) Flow velocity evolution in the plasma beam center at a distance of $\sim 28 \mathrm{~mm}$ in front of the target. The plasma stagnation region is indicated by the grey patch. The legend refers to the plasma discharge number in table 1. (b) Illustration of the near-target ionization process and the resulting axial flow velocity distribution. The grey patch denotes the plasma stagnation region at the upstream position.

\subsection{Heat flux reduction}

Motivated by the near-target ionization process, near-target plasma cooling is proposed to explain the reduced peak heat flux. To directly prove this, additional time-resolved TS measurements at $\sim 7 \mathrm{~mm}$ in front of the target of discharge \#1-3 were performed. As shown in figure $6, T_{\mathrm{e}}$ at $x=7 \mathrm{~mm}$ is smaller than at $x=28 \mathrm{~mm}$ during the pulses. The $T_{\mathrm{e}}$ reduction between the two positions increases with the value of $T_{\mathrm{e}}$ at $28 \mathrm{~mm}$ and is up to $\sim 40 \%$. Conversely, an increase in $n_{\mathrm{e}}$ is observed except for discharge \#3, as shown in figure B. As the neutral density is higher near the target, a stronger electron temperature cooling is expected when further approaching the target. Future time-resolved TS measurements even closer to the target may be achieved by carefully suppressing the stray light from the target [31].

A simple analytical model is next used to estimate the average electron temperature near the target. For the locally selfsustained plasma, the following relationship between the average electron temperature $\bar{T}_{\mathrm{e}}^{\text {target }}$ and average neutral density $\bar{n}_{\mathrm{n}}$ holds [27]:

$$
\frac{\pi}{2}-1=K \frac{\langle\sigma v\rangle_{\mathrm{iz}}\left(\bar{T}_{\mathrm{e}}^{\mathrm{target}}, \bar{n}_{\mathrm{e}}^{\mathrm{target}}\right)}{c_{\mathrm{se}}\left(\bar{T}_{\mathrm{e}}^{\mathrm{target}}\right)} \bar{n}_{\mathrm{n}} L,
$$

where $\langle\sigma v\rangle_{\text {iz }}\left(\bar{T}_{\mathrm{e}}^{\text {target }}, \bar{n}_{\mathrm{e}}^{\text {target }}\right)$ is the electron impact ionization rate coefficient of hydrogen (extracted from the AMJUEL database [32]). $L$ is the distance from the ionization front in which $M=0$ (as measured by CTS in figure 5(a)) to the target, where $M=1$ (Bohm criterion). In our case, $L$ can be assumed to be the ionization mean-free-path $\left(\lambda_{\mathrm{iz}}\right) . K$ is a scaling factor to incorporate the uncertainties of the right-hand side (RHS) terms of equation (2), which will be discussed in figure 8 . The derivation of equation (2) is given in appendix $\mathrm{C}$.

Figure 7 illustrates the application of equation (2). The filled contour plot shows the $\langle\sigma v\rangle_{\text {iz }}$ distribution. $\lambda_{\text {iz }}$ is calculated by taking the relative velocity $v_{\text {rel }}$ between the ions and

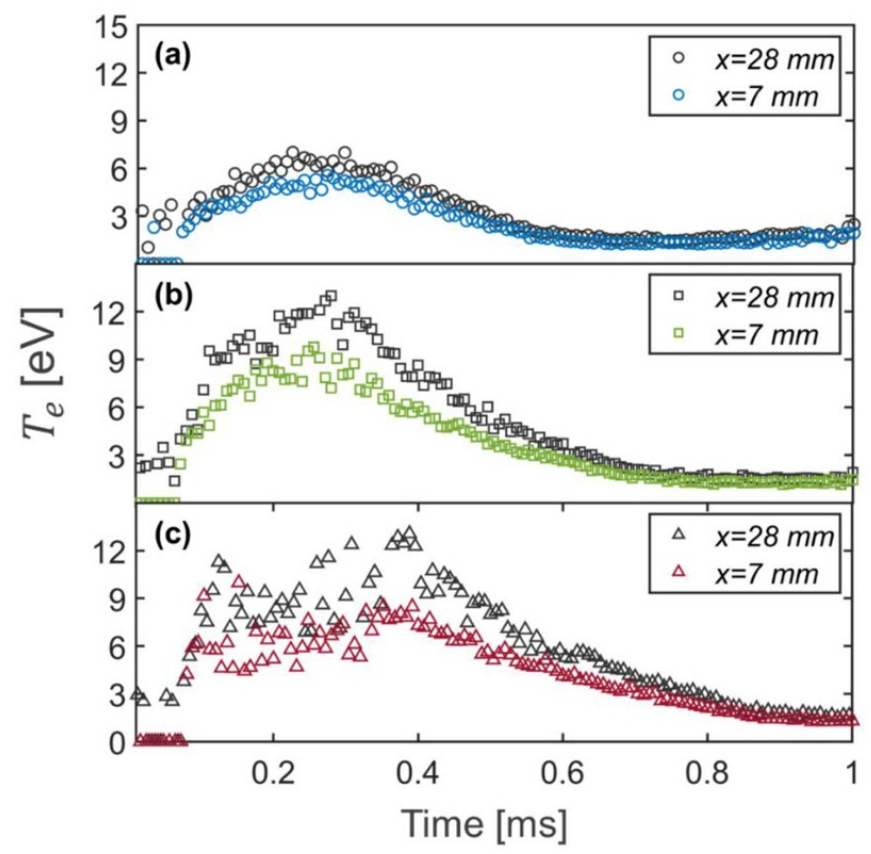

Figure 6. Observation of the near-target plasma cooling.

Time-resolved TS measurements at $\sim 7 \mathrm{~mm}$ and $\sim 28 \mathrm{~mm}$ in front of the target of (a) discharge \#1, (b) discharge \#2, and (c) discharge \#3.

the neutrals equal to the ion velocity at the sheath edge $c_{\mathrm{se}}$ (a sensitivity study of $v_{\text {rel }}$ will be shown in figure 8). If $\lambda_{\text {iz }}$ is larger the plasma beam size, ionization is unlikely to affect the plasma flow behavior. Therefore, equation (2) applies to the region where $\lambda_{\mathrm{iz}}$ is smaller than the plasma beam size, which is approximately $10 \mathrm{~mm}$ in this study. As an example, the timeresolved TS data of discharge \#2 (at $x=28 \mathrm{~mm}$ ) are shown in the plot through the green symbols whereby the pulse evolution direction is indicated by the purple arrow. The filled symbols correspond to $\lambda_{\mathrm{iz}}<10 \mathrm{~mm}$ and are therefore influenced by ionization. Then, equation (2) is plotted by setting $L=\lambda_{\mathrm{iz}}$ and 


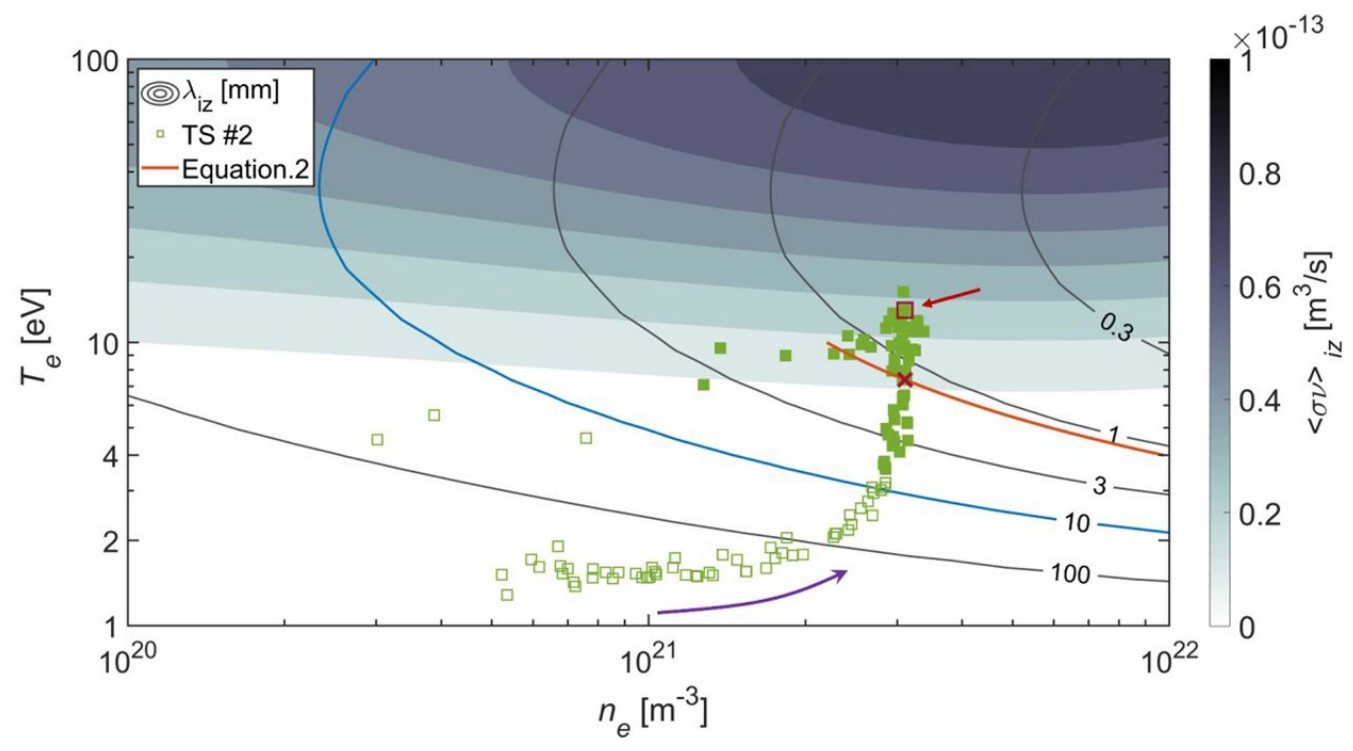

Figure 7. Calculation of the near-target electron temperature. The solid red square shows the peak $T_{\mathrm{e}}^{\mathrm{TS}}$ whereas the red cross shows the peak $\bar{T}_{\mathrm{e}}^{\text {target }}$ calculated by equation (2). The filled symbols correspond to $\lambda_{\mathrm{iz}}<10 \mathrm{~mm}$ (which is approximately the plasma beam size in this study) and are therefore affected by ionization. The purple arrow indicates the plasma pulse evolution direction of discharge \#2 at $x=28 \mathrm{~mm}$.
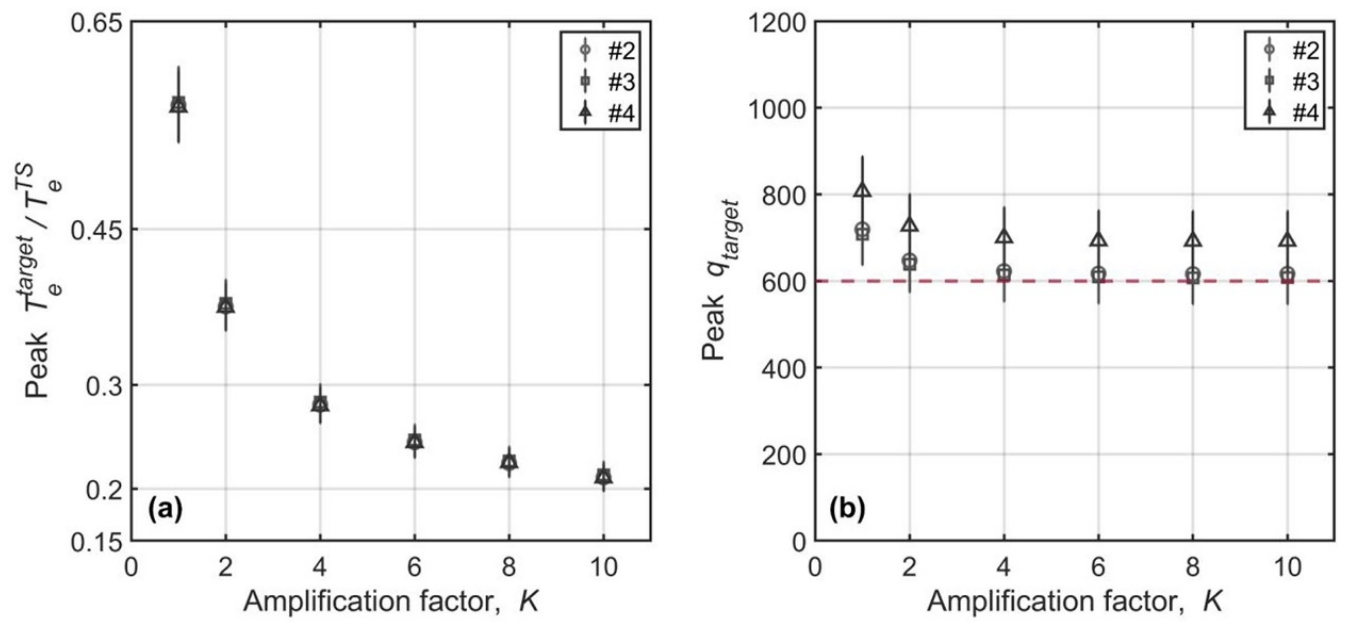

Figure 8. (a) $\frac{\bar{T}_{\mathrm{e}}^{\text {target }}}{T_{\mathrm{T}}^{\mathrm{TS}}}$ vs. the amplification factor $K . \bar{T}_{\mathrm{e}}^{\text {target }}$ is the calculated electron temperature near the target using equation (2) and $T_{\mathrm{e}}^{\mathrm{TS}}$ is the measured electron temperature at the TS position $(x=28 \mathrm{~mm})$. (b) Peak $q_{\text {target }}$ vs. the amplification factor $K$. $q_{\text {target }}$ is calculated using equation (1) with $\bar{T}_{\mathrm{e}}^{\text {target }}$ in (a). The red line denotes the corresponding FEM results in figure 3(d).

$\bar{n}_{\mathrm{n}}=\bar{n}_{\mathrm{e}}^{\text {target }}=n_{\mathrm{e}}^{\mathrm{TS}}$ of the peak electron temperature $T_{\mathrm{e}}^{\mathrm{TS}}$ which is highlighted in red (indicated by the red arrow; the largest $T_{\mathrm{e}}$ maybe an outlier and is therefore ignored). The intersection of the TS profile and equation (2) then yields $\bar{T}_{\mathrm{e}}^{\text {target }}$, as denoted by the red cross. This exercise is repeated for discharge \#3 and \#4. The results, expressed as $\frac{\bar{T}_{\mathrm{e}}^{\text {target }}}{T_{\mathrm{e}}^{\mathrm{TS}}}$, are shown in the most left symbol in figure $8(\mathrm{a})$.

The uncertainty of equation (2) is discussed next. On the RHS of equation (2), $L$ is subjected to the uncertainty in $v_{\text {rel }}$. Moreover, $\bar{n}_{\mathrm{n}}$ and $\bar{n}_{\mathrm{e}}^{\text {target }}$ can be larger than $n_{\mathrm{e}}^{\mathrm{TS}}$ due to particle recycling. For example, the peak $n_{\mathrm{e}}$ at the divertor is about $450 \%$ of the inter-ELM value in the DIII-D tokamak [12]. In Magnum-PSI, a global model suggests that the neutral flux can be amplified by a factor of $\sim 10$ [22]. Therefore, an amplification factor $K$ is introduced to incorporate the combined uncertainties of the RHS terms of equation (2). A sensitivity study of $K$ is shown in figure 8. As expected, the larger the value of $K$, the smaller $\frac{\bar{T}_{\mathrm{e}}^{\text {target }}}{T_{\mathrm{e}}^{\mathrm{TS}}}$. Likewise, $q_{\text {target }}$ vs. $K$ is shown in figure $8(\mathrm{~b})$. In calculating $q_{\mathrm{target}}, \bar{n}_{\mathrm{e}}^{\text {target }}$ is found by assuming a plasma pressure balance between the near-target ionization layer (assuming an average flow velocity of $0.5 \mathrm{Mach}$ ) and the plasma at the TS position. Pressure loss due to elastic collisions is thus not considered, which is not expected to be strong in the middle of the plasma pulses where the $T_{\mathrm{e}}$ is high $(>10 \mathrm{eV}) . q_{\text {target }}$ has a weak dependence on $K$, which approaches $600-700 \mathrm{MW} \mathrm{m}^{-2}$ when $K>4$. This is close to the values determined by FEM in figure 3(d) (as indicated by the red line) and therefore supports the hypothesis that the peak heat flux reduction is due to near-target plasma cooling. 

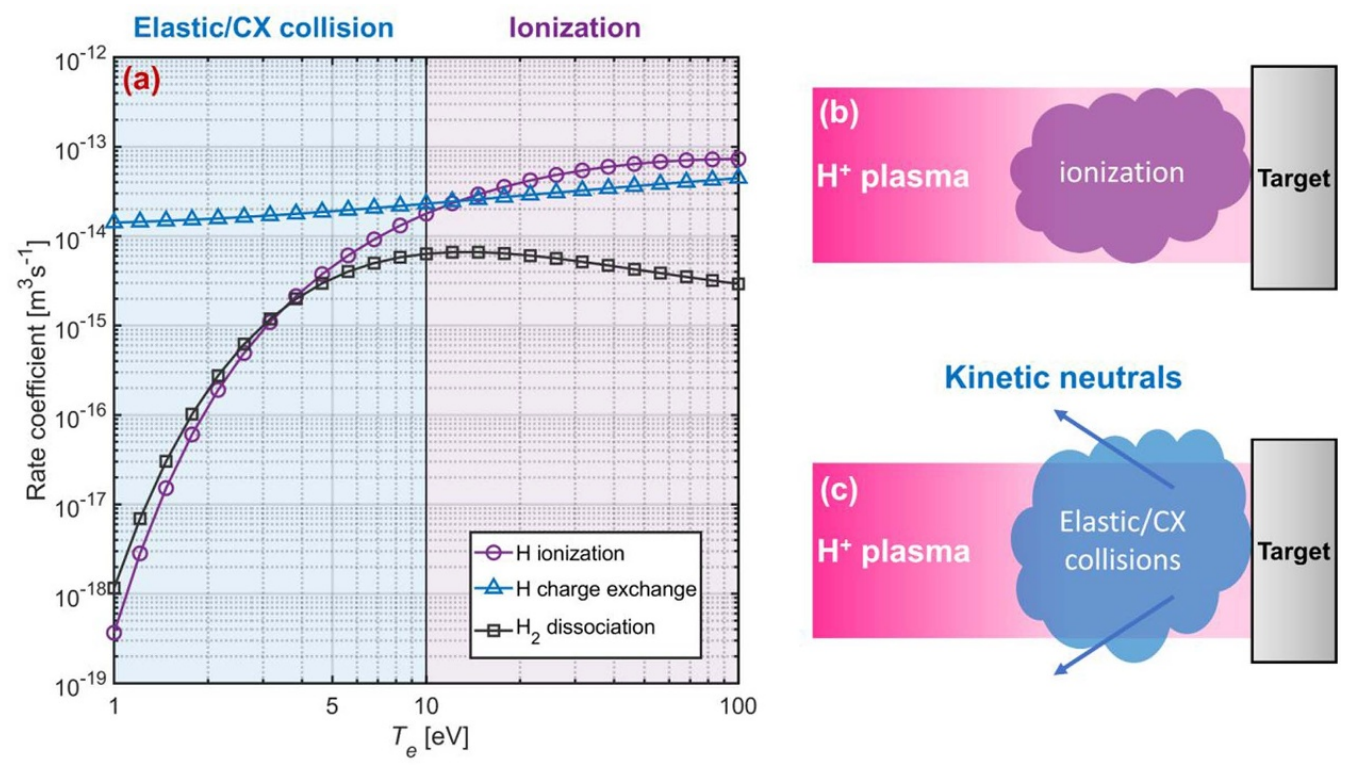

Figure 9. Energy dissipation mechanisms. (a) Rate coefficients of the indicated atomic reactions. (b) Ionization dominated plasma-neutral interactions, partially forming a near-target self-sustained plasma. (c) Elastic/CX collisions dominated plasma-neutral interactions.

At this point, it is worth discussing the global power balance. Clearly, the convective heat flux is negligible at the upstream position when the plasma is stagnant. Using the electron temperatures at the two positions in figure 6 and assuming an electron heat conductivity $\kappa_{0 \mathrm{e}}$ of 2000 [27], the peak conductive heat flux is estimated to be $\sim 200 \mathrm{MW} \mathrm{m}^{-2}$, which is only $\sim 30 \%$ of the corresponding heat flux absorbed by the target $\left(\sim 600 \mathrm{MW} \mathrm{m}^{-2}\right.$, see figure $\left.3(\mathrm{~d})\right)$. In Magnum-PSI, it has been shown that significant electric fields penetrate the plasma beam outside of the plasma source [33]. Therefore, the power deficiency may be provided by the Ohmic heating of these electric fields. However, this needs to be investigated in greater detail in future work. Moreover, in deriving equation (2), a uniform $T_{\mathrm{e}}$ in the near-target ionization layer is assumed. In reality, a temperature gradient may exist, which constitutes an additional conductive heat flux to the target. However, based on the heat flux comparison in figure 8(b), this temperature gradient is not expected to be strong.

\subsection{Energy dissipation mechanisms}

The energy dissipation in figure 4(b) is discussed next. Figure 9(a) plots the rate coefficients of three relevant atomistic physics processes for hydrogen as a function of $T_{\mathrm{e}}$ for $n_{\mathrm{e}}=2 \times 10^{21} \mathrm{~m}^{-3}$ and a relative energy between ions and neutrals of $3 \mathrm{eV}$ [32]. Varying $n_{\mathrm{e}}$ in the range of $10^{21}$ $5 \times 10^{21} \mathrm{~m}^{-3}$ only has a minor effect on the above rate coefficients. The number $N$ of elastic/charge exchange (elast$\mathrm{ic} / \mathrm{CX}$ ) collisions a recycling neutral undergoes before ionization when entering an infinite plasma at constant $n_{\mathrm{e}}$ and $T_{\mathrm{e}}$ is estimated as [29]: where the ionization rate $\langle\sigma v\rangle_{\mathrm{iz}}, \mathrm{CX}$ rate $\langle\sigma v\rangle_{\mathrm{CX}}$ and dissociation rate.

$\langle\sigma v\rangle_{\text {dis }}$ are plotted in figure 9(a). At $T_{\mathrm{e}}=10 \mathrm{eV}, N$ equals $\sim 0.4$. Therefore, when $T_{\mathrm{e}}>10 \mathrm{eV}$, ionization prevails. The $T_{\mathrm{e}}$ domain of ionization and elastic/CX collisions is highlighted by the colored patches in figure 9(a). As explained earlier, the near-target ionization process converts the upstream plasma into a plasma with a decreasing electron temperature and an increasing density near the target (figure 9(b)), entailing a heat flux reduction. However, ionization does not lead to significant energy loss because the energy removed by neutrals is subsequently re-deposited onto the target ${ }^{5}$.

When $T_{\mathrm{e}}<10 \mathrm{eV}$, elastic/CX collisionsf between the plasma ions and recycling neutrals becomes important. In this case, a large fraction of the neutrals escapes the plasma beam, taking away momentum and energy from the plasma (figure 9(c)). For a hydrogen plasma, the power removal by neutrals can be estimated as described in [29]:

$$
\frac{P(\text { coll. })}{P(\text { no coll. })}=\frac{M(5.2-\ln M)+\left(\frac{2}{\pi}\right)^{\frac{1}{2}}\left(\frac{T_{\mathrm{i}}}{T_{\mathrm{e}}}\right)^{\frac{3}{2}}}{\left(5.2+2 \frac{T_{\mathrm{i}}}{T_{\mathrm{e}}}\right)\left(1+\frac{T_{\mathrm{i}}}{T_{\mathrm{e}}}\right)^{\frac{1}{2}}},
$$

where $P$ (coll.) and $P$ (no coll.) are the plasma power with and without elastic/CX collisions. $M$ is the upstream plasma flow Mach number resulting from elastic/CX collisions. The largest Mach number $(M=0.2)$ in figure 5(a), for which $T_{\mathrm{e}}$ is small, mostly results from elastic/CX collisions. $M=0.2$ has also been measured for a steady-state hydrogen plasma in Magnum-PSI previously [22]. Using $M=0.2$ and $\frac{T_{\mathrm{i}}}{T_{\mathrm{e}}}=1$

\footnotetext{
5 Note that for a transient heat process, a reduction in heat flux does not neces-

sarily entail an energy loss.
}

$$
N \propto \frac{\left\langle\sigma v>_{\mathrm{CX}}\right.}{\left\langle\sigma v>_{\mathrm{iz}}\right.} \frac{\left\langle\sigma v>_{\mathrm{dis}}\right.}{\left\langle\sigma v>_{\mathrm{iz}}\right.}
$$


in equation (4), we obtain $\frac{P(\text { coll. })}{P(\text { no coll. })}=0.21$. Because elastic/CX collisions only dominate part of the plasma pulse, 0.21 is therefore the minimum value of $\frac{\eta_{\text {target }}}{\eta_{\text {collisionless }}}$ that can be attributed to elastic/CX collisions. This analysis implies that elastic/CX collisions constitute an important energy-loss mechanism in this study. Note that electron cooling is not considered in equation (4), the influence of which requires future effort.

\subsection{Implications for ITER}

The results described here may be highly relevant for the understanding of ELM induced heat loads on the tungsten PFCs in ITER. Figure 4 shows that the higher the electron density $n_{\mathrm{e}}$, the higher the energy loss. This energy-loss dependence on $n_{\mathrm{e}}$ can be rationalized as follows. First, the higher the $n_{\mathrm{e}}$, the smaller the electron temperature $T_{\mathrm{e}}$ as a result of increased ionization (figure 8). Then, the lower the $T_{\mathrm{e}}$ the smaller the mean-free-path of the elastic/CX collisions. Therefore, more collisions can occur inside the plasma beam, entailing an energy loss of the plasma. Currently, the main method to predict heat fluxes onto the target during ELMs in tokamaks is called the free-streaming-particle approach $[34,35]$. However, this approach does not fully consider the influence of particle recycling on the heat loads due to ELMs. If a higher $n_{\mathrm{e}}$ were to occur in ITER, e.g. in the presence of detachment, the here reported heat flux reduction and energy loss by near-surface plasma-neutral interactions can be important.

The $T_{\mathrm{e}}$ reduction is also beneficial for the operation of fusion reactors. A reason to choose tungsten as the PFC material is because of its low physical sputtering yield. However, during ELMs, the ion impact energy can be a few keV [11]. The erosion of the tungsten PFCs by ELMs is therefore a concern [36]. On the other hand, the core plasma has a low tolerability to tungsten impurities, as tungsten degrades the core plasma performance [37]. A reduction of $T_{\mathrm{e}}$ therefore extends the lifetime of PFCs and improves the core plasma performance.

We note that the electron (ion) temperature of ELM-like plasma in this study is different from those in tokamaks (a dozen or so $\mathrm{eV}$ vs. hundreds of $\mathrm{eVs}$ to thousands of $\mathrm{eVs}$ ). This could change the power deposition behavior of ELMs on the target. A higher electron temperature is expected to facilitate the ionization of recycling neutrals, entailing a stronger electron cooling than in the current study. However, if the electron temperature (after cooling by ionization) is still too high to enter the regime of elastic/CX collisions (figure 9), less energy dissipation (by elastic/CX collisions) is expected than in the current study. For experimental and numerical research devoted to tokamaks, the readers are referred to $[5,7,11,12,38,39]$. Nevertheless, as discussed earlier, these studies do not fully consider the influence of particle recycling on the heat loads induced by ELMs. Additionally, ELMs in future fusion devices are expected to occur with a high plasma density compared to current-day devices. The results reported here are therefore complementary to these studies.

\section{Conclusions}

In summary, with the aid of time-resolved TS, CTS and fast infrared thermography, we have studied the power deposition behavior of ELM-like hydrogen plasmas on tungsten in detail. An unprecedented plasma density regime was explored, and the major findings are:

(a) Through the comparison of heat fluxes between the target and a conventional collisionless sheath model (using the $n_{\mathrm{e}}$ and $T_{\mathrm{e}}$ measured $\sim 28 \mathrm{~mm}$ in front of the target), we reveal an inconsistency of the peak heat flux of up to $\sim 50 \%$ and of the energy fluence of up to $\sim 70 \%$. The latter exhibits a positive correlation with the peak $n_{e}$.

(b) From CTS, a plasma flow velocity as small as $0.03 \pm 0.02$ Mach is measured at $\sim 28 \mathrm{~mm}$ in front of the target. By varying the TS position from $\sim 28 \mathrm{~mm}$ to $\sim 7 \mathrm{~mm}$ in front of the target, a $T_{\mathrm{e}}$ cooling of up to $\sim 40 \%$ is observed.

(c) An analytical model is used to estimate the near-target electron temperature when the ionization of recycling neutrals is predominant, suggesting a $T_{\mathrm{e}}$ cooling of up to $\sim 20 \%$ from $\sim 28 \mathrm{~mm}$ in front of the target. The impact of near-target plasma ionization on the plasma flow behavior and heat flux has been discussed.

(d) The elastic collisions between the plasma ions and recycling neutrals contribute to the observed energy fluence inconsistency, based on the estimation of the power removal by the former and the $n_{\mathrm{e}}$ dependence of the latter.

Note that only one-dimensional models were used to analyze the plasma transport behavior in the innermost flux tube of the plasma beam. For a more thorough interpretation of the results, advanced numerical tools such as B2.5-Eunomia [40, 41], particle-in-cell [7, 42] and Vlasov [43, 44] are recommended for future work.

\section{Data availability statement}

The data that support the findings of this study are available upon reasonable request from the authors.

\section{Acknowledgments}

We acknowledge the support of the Magnum-PSI Facility Team at DIFFER. The Magnum-PSI facility at DIFFER has been funded by the Netherlands Organisation for Scientific Research (NWO) and EURATOM. This work was supported by the European Commission and carried out within the framework of the Erasmus Mundus International Doctoral College in Fusion Science and Engineering (FUSION-DC). This work has been carried out within the framework of the EUROfusion Consortium and has received funding from the Euratom research and training programme 2014-2018 and 2019-2020 under Grant Agreement No. 633053. The views and opinions expressed herein do not necessarily reflect those of the ITER Organization or of the European Commission. 


\section{Appendix A. Sheath heat transmission coefficient $\gamma$ of $\mathrm{H}^{+}$on tungsten}

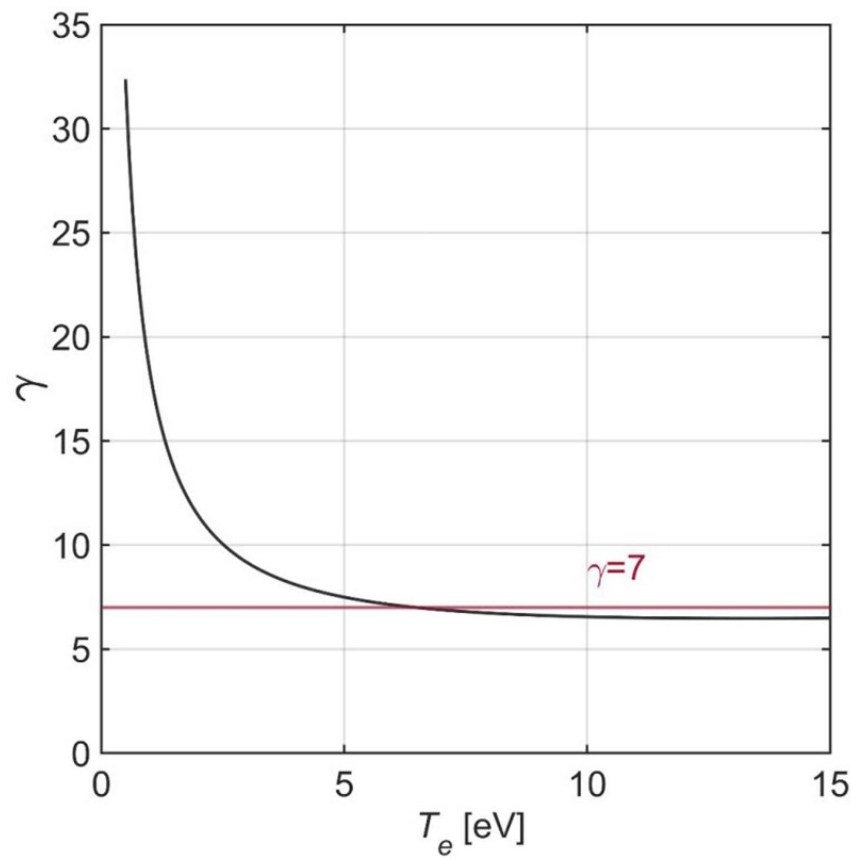

Figure A. The calculated $\gamma$ vs. $T_{\mathrm{e}}$ for $\mathrm{H}^{+}$on tungsten.

The sheath heat transmission coefficient $\gamma$ takes the following expression [27],

$$
\begin{aligned}
\gamma= & \left(2.5-e V_{\mathrm{s}}-e V_{\mathrm{ps}}\right)\left(1-R_{\mathrm{i}, \mathrm{E}}\right)+\frac{\chi_{\mathrm{i}}}{k_{\mathrm{B}} T_{\mathrm{e}}} \\
& +\frac{\chi_{\mathrm{r}}}{k_{\mathrm{B}} T_{\mathrm{e}}}\left(1-R_{\mathrm{i}, \mathrm{N}}\right)+\frac{2}{1-\delta}\left(1-R_{\mathrm{e}, \mathrm{E}}\right),
\end{aligned}
$$

in which the factor 2.5 in the first term accounts for the average fluid velocity of the ions at the sheath entrance. The energy gained in the potential drop of the pre-sheath and sheath are $e V_{\mathrm{ps}}=\ln 2$ and $e V_{\mathrm{s}}=\frac{1}{2} \ln \left[\left(2 \pi \frac{m_{\mathrm{e}}}{m_{\mathrm{i}}}\right)\left(1+\frac{T_{\mathrm{i}}}{T_{\mathrm{e}}}\right)(1-\delta)^{-2}\right]$, respectively. $R_{\mathrm{i}, \mathrm{E}}$ is the ion energy reflection coefficient $(\sim 0.5)$, taken from the TRIM.SP database [45] using a 3rd order polynomial extrapolation to lower energies. The second term represents the contribution from the electron-ion recombination, with $\chi_{\mathrm{i}}$ being $13.6 \mathrm{eV}$ for hydrogen. The third term refers to the atom-atom recombination energy $\chi_{\mathrm{r}}(2.2 \mathrm{eV}$ for hydrogen). Ion particle reflection $(\sim 0.7)$ is also allowed for by $R_{\mathrm{i}, \mathrm{N}}$, which is also taken from the TRIM.SP database with a 3rd order polynomial extrapolation to lower energies. Finally, the energy deposited by electrons is considered, where $R_{\mathrm{e}, \mathrm{E}}$ is the electron energy reflection coefficient and $\delta$ is the secondary electron emission yield. $\delta$ is calculated with the YoungDekker formula [46]. Due to the lack of a generalized form of $R_{\mathrm{e}, \mathrm{E}}$, it is fixed to the value 0.15 , which is well in the range of multiple experimental measurements [47]. In our previous analysis [10], $R_{\mathrm{i}, \mathrm{E}}, R_{\mathrm{i}, \mathrm{N}}, R_{\mathrm{e}, \mathrm{E}}$ and $\delta$ were fixed using values suggested in [27]. Here, we have refined these parameters, making them explicitly dependent on $T_{\mathrm{e}}$. This is expected to be more suitable for the pulsed plasmas. The derived $\gamma$ as a function of $T_{\mathrm{e}}$ is plotted in figure A. At a high $T_{\mathrm{e}}(>5 \mathrm{eV}), \gamma$ approaches the commonly cited 7 value.

\section{Appendix B. $n_{\mathrm{e}}$ profiles at $x=7 \mathrm{~mm}$}

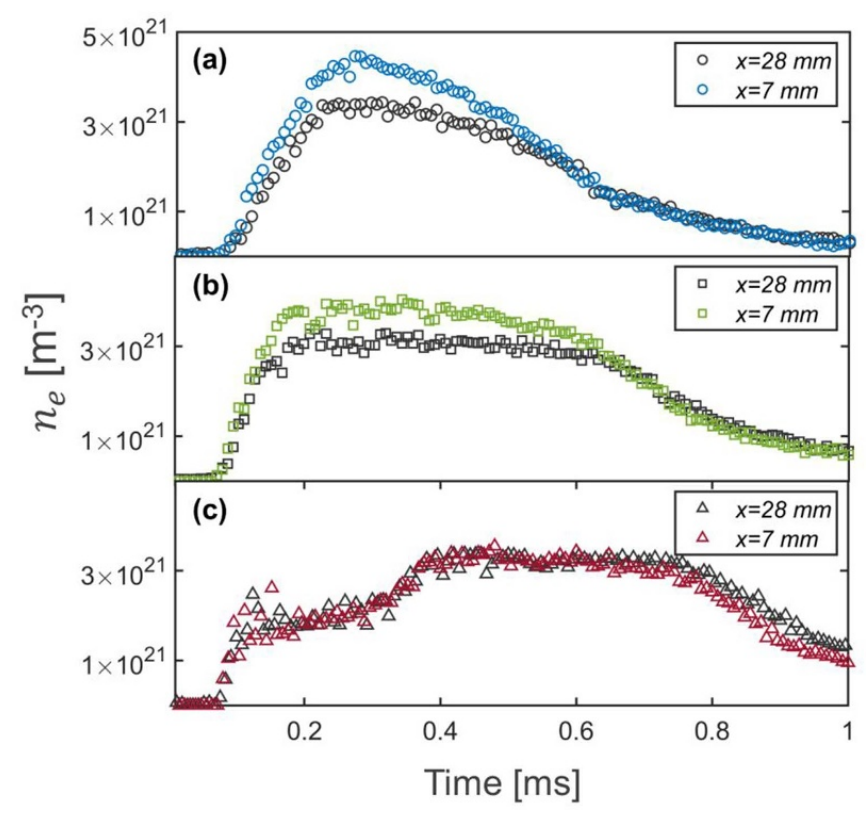

Figure B. $N_{\mathrm{e}}$ profiles of the same discharges in figure 6 at $x=7 \mathrm{~mm}$. (a) Discharge \#1, (b) discharge \#2, and (c) discharge \#3.

\section{Appendix C. Derivation of equation (2)}

This derivation closely follows chapter 1 of [27]. In 1-D, the particle and momentum conservation of ions yields:

$$
\frac{\mathrm{d} M}{\mathrm{~d} x}=\frac{S_{\mathrm{p}}}{n_{\mathrm{e}} c_{\mathrm{se}}} \frac{\left(1+M^{2}\right)}{\left(1-M^{2}\right)},
$$

where $M \equiv \frac{v}{c_{\mathrm{se}}}$ is the plasma Mach number, and $S_{\mathrm{p}} \equiv$ $n_{\mathrm{e}} n_{\mathrm{n}}\langle\sigma v\rangle_{\mathrm{iz}}$ is the ionization particle source. Integrating equation $(\mathrm{C} 1)$, one obtains:

$$
2 \tan ^{-1} M-M=\frac{n_{\mathrm{n}}\langle\sigma v\rangle_{\mathrm{iz}} x}{c_{\mathrm{se}}} .
$$

At the target $x=L, M(L)=1$ (Bohm's criterion) and at $x=0$, $M(0)=0$, so that equation $(\mathrm{C} 2)$ becomes:

$$
\frac{\pi}{2}-1=K \frac{\langle\sigma v\rangle_{\mathrm{iz}}\left(T_{\mathrm{e}}, n_{\mathrm{e}}\right)}{c_{\mathrm{se}}\left(T_{\mathrm{e}}\right)} \bar{n}_{\mathrm{n}} L .
$$

In the above derivation, it is assumed that $\sigma v_{\mathrm{iz}}\left(T_{\mathrm{e}}, n_{\mathrm{e}}\right)$ and $n_{\mathrm{n}}$ do not vary between 0 and $L$. This assumption is made because the $n_{\mathrm{n}}$ distribution cannot be obtained without extensive numerical computations. Therefore, the average quantities $\langle\sigma v\rangle_{\text {iz }}\left(T_{\mathrm{e}}, n_{\mathrm{e}}\right)$ and $\bar{n}_{\mathrm{n}}$ are used. When assuming that the neutral density decays exponentially from the target, a factor of $\left(1-\frac{1}{e}\right)$ should be added on the RHS of equation (C3). The influence of this factor and the uncertainty in $\bar{n}_{\mathrm{n}}$ and $L$ are taken into account by introducing a scaling factor $K$ on the RHS of equation (C3). Moreover, for simplicity, a uniform $T_{\mathrm{e}}$ is assumed in this near-target ionization layer. 


\section{ORCID iDs}

Y Li (D) https://orcid.org/0000-0002-9366-7474

T W Morgan (D) https://orcid.org/0000-0002-5066-015X J van den Berg-Stolp (D) https://orcid.org/0000-0002-11774373

G De Temmerman (D) https://orcid.org/0000-0002-41730961

J P M Hoefnagels (D) https://orcid.org/0000-0001-8359-7575

\section{References}

[1] Pitts R A et al 2019 Nucl. Mater. Energy 20100696

[2] De Temmerman G et al 2018 Plasma Phys. Control. Fusion 60044018

[3] Zohm H 1996 Plasma Phys. Control. Fusion 38105

[4] Ham C et al 2020 Nat. Rev. Phys. 2159

[5] Guillemaut C et al 2015 Plasma Phys. Control. Fusion 57085006

[6] Knolker M et al 2018 Nucl. Fusion 58096023

[7] Dai S et al 2018 Nucl. Fusion 58014006

[8] Van Eden G G et al 2014 Nucl. Fusion 54123010

[9] Wirtz M et al 2017 Nucl. Fusion 57066018

[10] Li Y et al 2020 Nucl. Fusion 60046029

[11] Eich T et al 2017 Nucl. Mater. Energy 1284

[12] Abrams T et al 2018 Nucl. Mater. Energy 17164

[13] Field A R et al 2017 Plasma Phys. Control. Fusion 59095003

[14] Zielinski J J et al 2014 Appl. Phys. Lett. 104124102

[15] Morgan T W et al 2014 Plasma Phys. Control. Fusion 56095004

[16] Van Rooij G J et al 2007 Appl. Phys. Lett. 90121501

[17] Van Eck H J N et al 2019 Fusion Eng. Des. 14226

[18] Temmerman G D et al 2011 Nucl. Fusion 51073008

[19] Van Eck H J N et al 2009 J. Appl. Phys. 105063307
[20] Van Der Meiden H J et al 2012 Rev. Sci. Instrum. 83123505

[21] Van Der Meiden H J et al 2016 Appl. Phys. Lett. 109261102

[22] Van Den Berg-Stolp J et al 2020

[23] Chen Z et al 2020 Nucl. Fusion 60046020

[24] Li Y et al 2021 Nucl. Fusion 61046018

[25] Ho C Y et al 1972 J. Phys. Chem. Ref. Data 1279

[26] White G K et al 1984 J. Phys. Chem. Ref. Data 131251

[27] Stangeby P C 2000 The Plasma Boundary of Magnetic Fusion Devices (London: Institute of Physics Publishing Bristol)

[28] Riemann K U 1991 J. Phys. D: Appl. Phys. 24493

[29] Stangeby P C 1993 Nucl. Fusion 331695

[30] Krasheninnikov S I et al 1997 Phys. Plasmas 41638

[31] Van Den Berg J et al 2019 J. Instrum. 14 C10041

[32] Reiter D 2000 Electronic file, FZ Jülich (https://doi.org/ 10.1258/1357633001934528)

[33] Shumack A E et al 2012 Plasma Phys. Control. Fusion 54125006

[34] Fundamenski W et al 2006 Plasma Phys. Control. Fusion 48109

[35] Eich T et al 2009 J. Nucl. Mater. 390-391 760

[36] Abrams T et al 2019 Phys. Plasmas 26062504

[37] Federici G et al 2001 Nucl. Fusion 411967

[38] Guillemaut C et al 2018 Nucl. Fusion 58066006

[39] Lunt T et al 2020 Plasma Phys. Control. Fusion 62105016

[40] Wieggers R C et al 2013 J. Nucl. Mater. 438 S643

[41] Perillo R et al 2019 Phys. Plasmas 26102502

[42] Tskhakaya D, Jachmich S, Eich T and Fundamenski W 2011 J. Nucl. Mater. 415 S860

[43] Moulton D et al 2013 Plasma Phys. Control. Fusion 55085003

[44] Coulette D et al 2016 Plasma Phys. Control. Fusion 58085004

[45] Eckstein W 2002 Calculated Sputtering, Reflection and Range Values (IPP 9/132)

[46] Tolias P 2014 Plasma Phys. Control. Fusion 56123002

[47] Tolias P 2016 (arXiv:1601.02047) [physics.plasm-ph] 